\title{
Correlation Between Nitric Oxide and Urodynamics in Men With Bladder Outlet Obstruction
}

\author{
Kang Jun Cho ${ }^{1}$, Jun Sung Koh ${ }^{1}$, Jin Bong Choi ${ }^{1}$, Sang Hi Park ${ }^{2}$, Weon Sun Lee ${ }^{2}$ Joon Chul Kim \\ ${ }^{1}$ Department of Urology, Bucheon St. Mary's Hospital, College of Medicine, The Catholic University of Korea, Seoul, Korea \\ ${ }_{2}^{2}$ Institute of Clinical Medicine Research, The Catholic University of Korea, Bucheon St. Mary's Hospital, Bucheon, Korea
}

\begin{abstract}
Purpose: To investigate the correlation between nitric oxide (NO) and urodynamics in men with bladder outlet obstruction (BOO) by analyzing nitric oxide synthase (NOS) in the urothelium.

Methods: We prospectively enrolled 25 men who planned to undergo surgical treatment for benign prostatic obstruction and identified as $\mathrm{BOO}$ in the preoperative urodynamics. Bladder tissue was taken during surgical prostate resection. Expressions of endothelial NOS (eNOS), inducible NOS (iNOS), and neuronal NOS (nNOS) in the urothelium were analyzed, and their correlation with urodynamic parameters was also assessed in all patients. We also compared the expressions of eNOS, iNOS, and $\mathrm{nNOS}$ between $\mathrm{BOO}$ with detrusor underactivity (DU) group and $\mathrm{BOO}$ without $\mathrm{DU}$ group.

Results: In all patients, the level of eNOS positively correlated with maximal flow rate and with maximum cystometric capacity (MCC). The level of iNOS positively correlated with MCC. nNOS levels were positively correlated with detrusor pressure at maximal flow and with bladder contractility index in all patients. The level of eNOS, iNOS, and nNOS did not significantly differ between $\mathrm{BOO}$ without DU group and $\mathrm{BOO}$ with DU group.

Conclusions: This study suggests that NO was correlated with bladder dysfunction in men with BOO. Particularly, nNOS may reflect the change in detrusor function.
\end{abstract}

Keywords: Nitric oxide synthase; Prostatic hyperplasia; Urinary bladder outlet obstruction; Urodynamics

- Funding Support: This study was supported by the Institute of Clinical Medicine Research of Bucheon St. Mary's Hospital, Research Fund.

- Research Ethics: This study was performed in accordance with the guidelines of the Declaration of Helsinki, and approved by the ethics committee and the institutional review board at our center (HIRB-20170925-035). All patients provided written informed consent before enrollment.

- Conflict of Interest: No potential conflict of interest relevant to this article was reported.

\section{INTRODUCTION}

Bladder outlet obstruction (BOO), which is characterized by reduced uroflow rate and increased residual urine volume despite increased detrusor pressure, can cause morphological and functional changes to the bladder, resulting in either an underactive bladder or an overactive bladder [1,2]. Patients with be- nign prostatic obstruction (BPO), which is one of the most common causes of $\mathrm{BOO}$ in men, experience not only voiding symptoms but also storage symptoms that can have a considerable negative impact on the quality of life. Moreover, BOO-induced bladder function changes can be related to the lack of improvements in subjective or objective findings even after surgical treatment for BPO [3]. At present, the most reliable diag-

Corresponding author: Joon Chul Kim (D) https://orcid.org/0000-0002-4019-620X Department of Urology, Bucheon St. Mary's Hospital, College of Medicine, The Catholic University of Korea, 327 Sosa-ro, Bucheon 14647, Korea Email: kjc@catholic.ac.kr

Submitted: December 7, 2021 / Accepted after revision: February 16, 2022 
nostic method for bladder changes due to BOO is urodynamics. However, this method has some limitations, such as being invasive and time consuming. Although urodynamics is the only investigation to explore detrusor underactivity (DU) or detrusor overactivity (DO), the diagnostic criteria of them are various and uncertain yet. Thus, the development of new biomarkers is needed for a less invasive and more accurate diagnosis of bladder functional changes. For new biomarkers to be applied to the patients with lower urinary tract symptoms, they must be able to diagnose the patient's condition, reflecting the results of the current urodynamics.

Pathophysiological changes in the urothelium induced by $\mathrm{BOO}$ are receiving attention as a new biomarker for diagnosing bladder dysfunction [4]. The urothelium acts as a sensor of various stimuli and is able to release various neurotransmitters that would modulate detrusor contraction and relaxation [5]. Nitric oxide (NO), which is catalyzed by 3 different isoforms of NO synthase (NOS) (i.e., endothelial NOS [eNOS], inducible NOS [iNOS], and neuronal NOS [nNOS]), could act as a neurotransmitter in the urothelium and influence the bladder function. Several previous animal studies have shown that the NOS isoforms show changes depending on the status of bladder function, such as an increase in nNOS and eNOS expression in DO associated with $\mathrm{BOO}[6]$ or a decrease in eNOS expression in $\mathrm{BOO}$, which is responsible for bladder dysfunction [7]. However, the differences in the expression levels of NOS isoforms in the urothelium depending on bladder function remain unclear. The role of urothelial NO in bladder dysfunction has been mainly investigated in animal studies, and only a few human studies have been performed. In the present study, we aimed to investigate the correlation between $\mathrm{NO}$ and urodynamic parameters in men with BPO through the analysis of NOS isoforms in the urothelium.

\section{MATERIALS AND METHODS}

\section{Study Population and Design}

The study participants were a new set of 25 men aged 50-75 years old who were scheduled for surgical treatment for BPO, such as transurethral resection of the prostate or holmium laser enucleation of the prostate, and underwent preoperative urodynamics. Urodynamics was conducted in accordance with the Good Urodynamic Practice standards recommended by the International Continence Society [8]. Patients who were diagnosed with prostate cancer before or after surgery for BPO, and those with histories of prostatic and/or urethral surgery, acute urinary tract infection or indwelling bladder catheterization within 1month, interstitial cystitis/bladder pain syndrome, and lower urinary tract malignancy were excluded.

Bladder specimens were obtained using 3 cold cup biopsy from the posterior bladder wall during bladder filling of 150$200 \mathrm{~mL}$ just before surgery. The specimens were frozen in liquid nitrogen until use in the analysis. The expression of eNOS, iNOS, and nNOS in the urothelium was evaluated using enzyme-linked immunosorbent assay (ELISA) and immunofluorescence staining. We investigated the correlations between the expression of each NOS isoform in the urothelium and the urodynamic parameters. We also compared the expression levels of each NOS isoform between patients with DU and those without DU. DU was defined as a bladder contractility index (BCI) of $<100$ in urodynamics.

\section{ELISA and Immunofluorescence Staining for NOS Isoforms} ELISA and immunofluorescence staining for the NOS isoforms were performed as described in our previous study [9]. The specimens were rinsed with $1 \times$ phosphate-buffered saline (PBS), homogenized in $500 \mu \mathrm{L}$ of $1 \times \mathrm{PBS}$, and stored overnight at $-20^{\circ} \mathrm{C}$. After 2 freeze-thaw cycles were conducted, the homogenates were centrifuged and the supernatant was removed. The levels of eNOS and iNOS in the specimens were measured, according to the manufacturer's instructions, using a human eNOS ELISA kit and a human iNOS ELISA kit (Cusabio Biotech LLC, Houston, TX, USA). The nNOS levels in the samples were measured using a human nNOS ELISA kit (MyBioSource Inc., San Diego, CA, USA). The optical density of each well was determined within 5 minutes using a microplate reader (VersaMax, Molecular Devices LLC, San Jose, CA, USA) set to 450 $\mathrm{nm}$ and $540 \mathrm{~nm}$.

For immunofluorescence analysis, we used antibodies against eNOS (1:100 dilution; Abcam Ltd., Cambridge, UK), nNOS (1:200; Abcam Ltd., UK), and iNOS (1:200; Thermo Fisher Scientific, Waltham, MA, USA). The slides were rinsed and then incubated with the secondary antibody (Alexa Fluor 488-labeled goat anti-rabbit IgG, 1:300; Molecular Probes, Eugene, OR, USA) at room temperature for 2 hours. After rinsing the samples again, the slides were stained with 4',6-diamidino2-phenylindole (Vector Laboratories, Burlingame, CA, USA), and examined using a fluorescence microscope (BX50; Olympus, Tokyo, Japan). 


\section{Statistical Analysis}

The sample size, calculated by the expected mean difference of 0.01 , the standard deviation of 0.008 , beta error of 0.2 , and alpha error of 0.05 , was planned to recruit 15 patients per county and a total of 30 patients, taking into account $10 \%$ dropout rates per county. All data are reported as means with standard error of means. The mean values were statistically compared using the Mann-Whitney U-test for all continuous variables and Fisher exact test for categorical variables. We analyzed the correlations between each NOS isoform in the urothelium and the urodynamic parameters using Spearman correlation analysis. All statistical analyses were performed using IBM SPSS Statis- tics ver. 20.0 (IBM Co., Armonk, NY, USA). A P-value of $<0.05$ indicated a significant difference.

\section{RESULTS}

Baseline clinical characteristics and urodynamic parameters of the 25 men with a mean age of $67.5 \pm 4.6$ years, and the comparison between the $\mathrm{BOO}$ with DU group $(\mathrm{n}=10)$ and the $\mathrm{BOO}$ without DU group $(\mathrm{n}=15)$ are presented in the Table 1. Clinical characteristics such as age, prostate volume, and symptom scores were not different significantly between the 2 groups. BOO with DU group had larger maximum cystometric capaci-

Table 1. Baseline characteristics of enrolled patients

\begin{tabular}{lcccc}
\hline Variable & $\begin{array}{c}\text { Total } \\
(\mathrm{n}=25)\end{array}$ & $\begin{array}{c}\text { BOO with DU } \\
\text { group }(\mathrm{n}=10)\end{array}$ & $\begin{array}{c}\text { BOO without DU } \\
\text { group }(\mathrm{n}=15)\end{array}$ & P-value \\
\hline Age $(\mathrm{yr})$ & $67.5 \pm 4.6$ & $68.8 \pm 4.3$ & $66.7 \pm 4.8$ & 0.261 \\
BMI $\left(\mathrm{kg} / \mathrm{m}^{2}\right)$ & $25.1 \pm 3.9$ & $25.3 \pm 2.9$ & $24.8 \pm 4.6$ & 0.397 \\
PSA $(\mathrm{ng} / \mathrm{mL})$ & $3.64 \pm 2.89$ & $2.53 \pm 1.75$ & $4.37 \pm 3.31$ & 0.144 \\
Total prostate volume $(\mathrm{mL})$ & $59.6 \pm 23.2$ & $52.4 \pm 19.7$ & $64.4 \pm 24.8$ & 0.196 \\
Transitional zone volume $(\mathrm{mL})$ & $31.2 \pm 15.4$ & $28.4 \pm 14.8$ & $33.0 \pm 16.1$ & 0.439 \\
IPSS total & $16.2 \pm 8.8$ & $14.4 \pm 7.7$ & $17.6 \pm 9.6$ & 0.285 \\
IPSS storage subscore & $5.9 \pm 3.7$ & $5.0 \pm 3.1$ & $6.6 \pm 4.1$ & 0.312 \\
IPSS voiding subscore & $10.3 \pm 5.6$ & $9.4 \pm 5.2$ & $10.9 \pm 6.0$ & 0.437 \\
IPSS QoL score & $3.4 \pm 1.1$ & $3.1 \pm 1.1$ & $3.7 \pm 1.0$ & 0.343 \\
Urodynamic parameter & & & & 0.071 \\
Qmax $(\mathrm{mL} / \mathrm{sec})$ & $7.1 \pm 3.5$ & $5.4 \pm 2.1$ & $8.3 \pm 3.9$ & 0.726 \\
Voided volume $(\mathrm{mL})$ & $219.4 \pm 77.8$ & $224.3 \pm 90.4$ & $216.4 \pm 72.4$ & 0.091 \\
PVR $(\mathrm{mL})$ & $167.3 \pm 110.9$ & $221.3 \pm 133.6$ & $131.4 \pm 78.4$ & 0.031 \\
MCC $(\mathrm{mL})$ & $375.9 \pm 88.6$ & $424.1 \pm 94.6$ & $343.8 \pm 70.2$ & 0.938 \\
MUCP $\left(\mathrm{cm} \mathrm{H}_{2} \mathrm{O}\right)$ & $70.9 \pm 39.1$ & $70.0 \pm 18.9$ & $71.3 \pm 47.4$ & $<0.001^{*}$ \\
PdetQmax $\left(\mathrm{cm} \mathrm{H}_{2} \mathrm{O}\right)$ & $61.6 \pm 21.6$ & $43.2 \pm 12.5$ & $73.9 \pm 17.3$ & $0.010^{*}$ \\
BOOI & $47.4 \pm 22.7$ & $32.5 \pm 13.9$ & $57.2 \pm 22.4$ & $0.012^{*}$ \\
BOO grade & & & & $<0.001^{*}$ \\
2-3 & $15(60.0)$ & $9(90.0)$ & $6(40.0)$ & $9(60.0)$ \\
4-6 & $10(40.0)$ & $1(10.0)$ & $115.5 \pm 18.3$ & \\
BCI & $97.3 \pm 28.1$ & $70.1 \pm 14.4$ & & \\
\hline
\end{tabular}

Values are presented as mean \pm standard error of the mean or number (\%).

BOO, bladder outlet obstruction; DU, detrusor underactivity; BMI, body mass index; PSA, prostate-specific antigen; IPSS, International Prostate Symptom Score; QoL, quality of life; Qmax, maximum flow rate; PVR, postvoid residual; MCC, maximum cystometric capacity; MUCP, maximal urethral closure pressure; PdetQmax, detrusor pressure on maximum flow; BOOI, bladder outlet obstruction index; BCI, bladder contractility index.

${ }^{\star} \mathrm{P}<0.05$, statistically significant difference. 
ty (MCC), lower BCI and lower $\mathrm{BOO}$ grade than those in the $\mathrm{BOO}$ without DU group but maximum flow rate (Qmax) and postvoid residual urine volume were not different between the 2 groups.

The eNOS level positively correlated with the $\mathrm{Qmax}(\mathrm{r}=0.499$, $\mathrm{P}=0.021)$ and with MCC $(\mathrm{r}=0.548, \mathrm{P}=0.006)$. The iNOS level positively correlated with MCC only $(r=0.483, \mathrm{P}=0.017)$. The nNOS level positively correlated with the detrusor pressure at $\operatorname{Qmax}(\mathrm{r}=0.626, \mathrm{P}=0.048)$ and with $\mathrm{BCI}(\mathrm{r}=0.609, \mathrm{P}=0.022)$

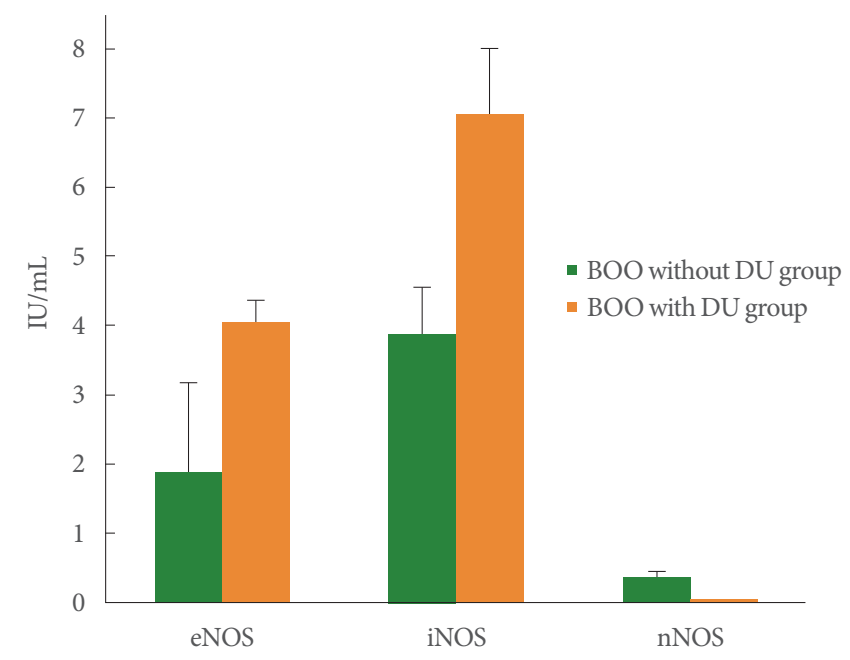

Fig. 1. Comparisons of eNOS, iNOS, and nNOS expression levels between the $\mathrm{BOO}$ with $\mathrm{DU}$ group and $\mathrm{BOO}$ without $\mathrm{DU}$ group. Data expressed as mean \pm standard error of the mean. eNOS, endothelial nitric oxide synthase; iNOS, inducible nitric oxide synthase; nNOS, neuronal nitric oxide synthase; $\mathrm{BOO}$, bladder outlet obstruction; DU, detrusor underactivity. in all patients (Table 2).

When the expression levels of each isoform of NOS were compared between the $\mathrm{BOO}$ with $\mathrm{DU}$ and $\mathrm{BOO}$ without $\mathrm{DU}$ groups, the levels of eNOS $(4.052 \pm 1.139$ vs. $1.863 \pm 1.316, \mathrm{P}=$ $0.073)$, iNOS (7.042 \pm 2.178 vs. $3.869 \pm 0.687, \mathrm{P}=0.347)$, and nNOS $(0.041 \pm 0.012$ vs. $0.351 \pm 0.096, \mathrm{P}=0.067)$ did not significantly differ between the 2 groups (Fig. 1). Immunofluorescence staining revealed that eNOS, iNOS, and nNOS were localized in the urothelium. However, no significant differences were found in the immunofluorescence signals evaluated using image analysis between the $\mathrm{BOO}$ without $\mathrm{DU}$ and $\mathrm{BOO}$ with DU groups (Fig. 2).

\section{DISCUSSION}

We identified the relationship between the urodynamic parameters in men with $\mathrm{BOO}$ and the expression of 3 different NOS isoforms that produce $\mathrm{NO}$ in the urothelium. The urothelium consolidates sensory information and reflects changes in bladder function. Thus, changes in neurotransmitters in the urothelium have been a focus of studies on new diagnostic markers for bladder dysfunction [10]. Moreover, a significant change in the expression of adenosine triphosphate in the urothelium of patients with DU was observed, on the basis of urodynamic parameters, although eNOS did not show a statistically significant relationship to detrusor function in our previous study [9]. Because we could not identify the change in eNOS with the change in detrusor function despite evidences that urothelial NO could reflect bladder dysfunction $[6,7,11]$, we attempted to investigate

Table 2. Correlation of eNOS, iNOS, and nNOS with urodynamic parameters

\begin{tabular}{|c|c|c|c|c|c|c|}
\hline \multirow{2}{*}{ Variable } & \multicolumn{2}{|c|}{ eNOS } & \multicolumn{2}{|c|}{ iNOS } & \multicolumn{2}{|c|}{ nNOS } \\
\hline & $\mathrm{r}$ & $\mathrm{P}$-value & $\mathrm{r}$ & $\mathrm{P}$-value & $\mathrm{r}$ & P-value \\
\hline Qmax & 0.499 & 0.021 & 0.405 & 0.068 & -0.126 & 0.788 \\
\hline VV & -0.084 & 0.716 & -0.221 & 0.336 & -0.679 & 0.094 \\
\hline PVR & -0.056 & 0.804 & -0.026 & 0.910 & 0.381 & 0.352 \\
\hline MCC & 0.548 & 0.006 & 0.483 & 0.017 & -0.624 & 0.054 \\
\hline MUCP & -0.428 & 0.068 & -0.433 & 0.064 & 0.262 & 0.531 \\
\hline PdetQmax & -0.369 & 0.076 & -0.209 & 0.327 & 0.626 & 0.048 \\
\hline $\mathrm{BCI}$ & -0.325 & 0.121 & -0.138 & 0.521 & 0.609 & 0.022 \\
\hline BOOI & -0.268 & 0.206 & -0.157 & 0.465 & 0.394 & 0.260 \\
\hline
\end{tabular}

eNOS, endothelial nitric oxide synthase; iNOS, inducible nitric oxide synthase; nNOS, neuronal nitric oxide synthase; Qmax, maximum flow rate; VV, voided volume; PVR, postvoid residual; MCC, maximal cystometric capacity; MUCP, maximal urethral closure pressure; PdetQmax, detrusor pressure on maximum flow; BCI, bladder contractility index; BOOI, bladder outlet obstruction index. 

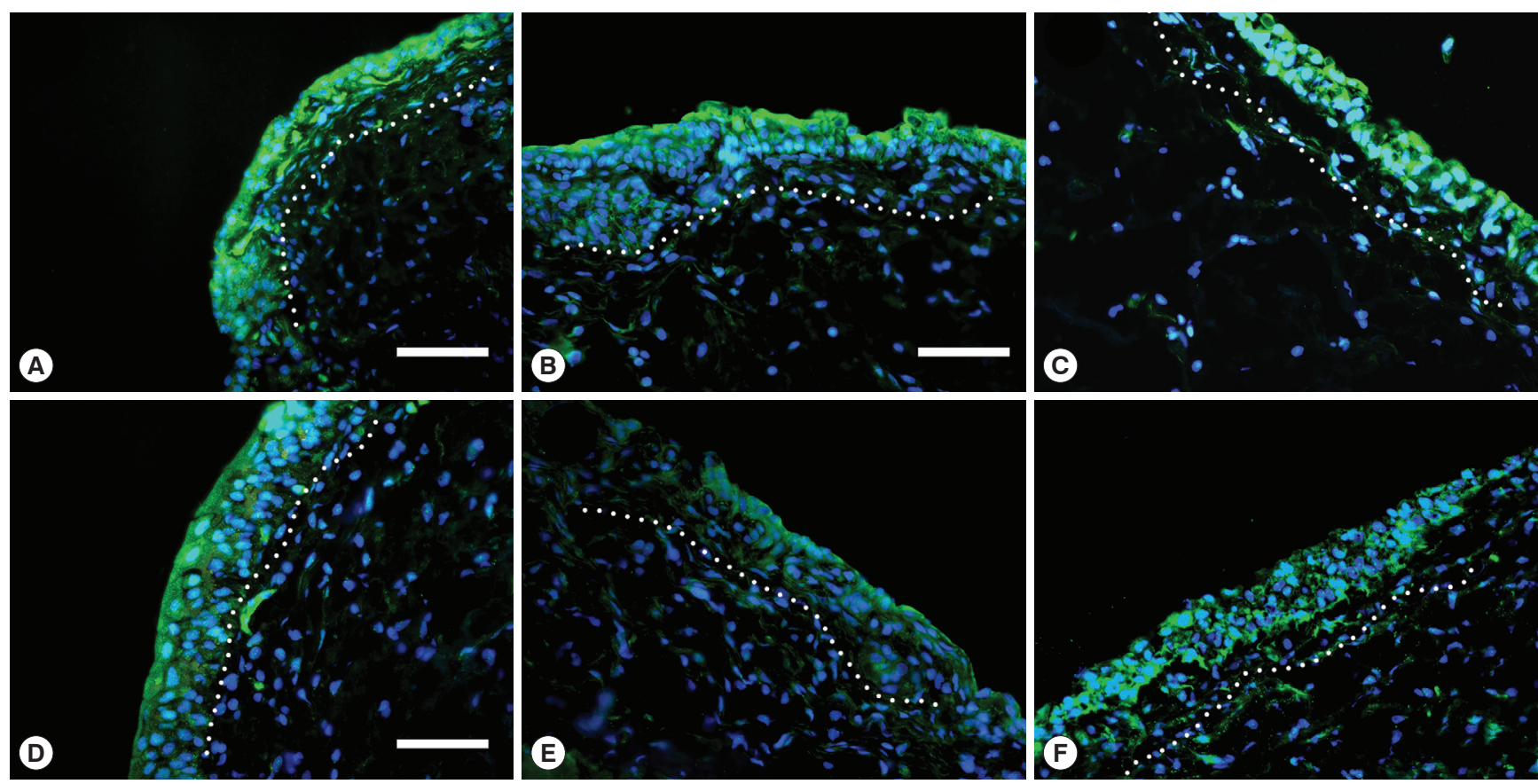

Fig. 2. Immunofluorescence staining of eNOS, iNOS, and nNOS. (A-C) eNOS, iNOS, nNOS expressions in the bladder tissues in the BOO with DU group, (D-F) eNOS, iNOS, nNOS expressions in the BOO without DU group. Scale bars: $50 \mu \mathrm{m}$, White dotted line separates urothelium from lamina propria. eNOS, endothelial nitric oxide synthase; iNOS, inducible nitric oxide synthase; nNOS, neuronal nitric oxide synthase; BOO, bladder outlet obstruction; DU, detrusor underactivity.

the relationship between $\mathrm{NO}$ and bladder function using all 3 NOS isoforms in a new set of patients. In the current investigation, we found that the level of eNOS positively correlated with Qmax and with MCC, and the level of iNOS positively correlated with MCC. The relation to Qmax or MCC alone cannot sufficiently reflect the change in bladder function. Although it is difficult to clearly explain the reasons for the correlations of eNOS with Qmax and MCC, which have not been reported in previous studies, they were likely caused by differences in the clinical characteristics of the enrolled patients, especially the symptom severity. Even though we have not directly compared the clinical characteristics in this study population with those of our previous study [9], there would be differences in urodynamics, such as the mean bladder outlet obstruction index, which was 47.4 in this study but it was less than 40 in our previous study. Further studies are needed to identify NOS isoform changes due to these clinical symptom differences.

Increased expression of eNOS in the presence of bladder hyperactivity related to the cyclophosphamide-induced cystitis was reported in an animal study [12], whereas decreased expression of eNOS in the presence of DU, which leads to decreased sensory proteins, was reported in a human study [13].
However, in our study in patients with DU, no significant differences in eNOS and iNOS expression were found.

One study showed that a decrease in iNOS was related to improvement of functional and fibrotic changes in the bladder of rats with BOO [14]. However, another study demonstrated that selective inhibition of iNOS was related to a decrease in bladder capacity, although it did not significantly decrease of bladder weight in mice with BOO. Conversely, the inhibition of all 3 NOS isoforms has been reported to increase nonvoiding contractions and to decrease the bladder capacity [15], thereby worsening the bladder function. Thus, only iNOS could not reflect bladder changes.

In this study, the expression of nNOS in the urothelium of men with BOO positively correlated with the detrusor pressure at Qmax and with BCI, which reflects bladder contractility. nNOS is one of the neurotransmitters released by afferent nerves in the bladder, and changes in nNOS suggest alterations in the afferent system [16]. Hu et al. [17] reported the change in nNOS-positive neurons according to the duration of partial $\mathrm{BOO}$ and change in bladder weight in an animal model. In another study, nNOS-positive neurons in partial BOO were decreased by NOS inhibitor administration and increased by L- 
arginine, which is a substrate for NO formation [18]. These findings suggest that changes in nNOS due to bladder morphological changes induced by $\mathrm{BOO}$ cannot sufficiently reflect the changes in nNOS resulting from changes in bladder function. Hannan et al. [19] observed a decrease in nerve terminals expressing nNOS at 1 month after injury in a rat model of bladder dysfunction induced by bilateral pelvic nerve injury, indicating that nNOS is related to impaired bladder function. Another study showed that NO derived from stimulated nNOS increased detrusor activity in the bladder of cats, by demonstrating increased time to onset of spontaneous detrusor contractions and decreased frequency of detrusor contractions due to nNOS inhibition [20]. This was a different effect compared with that of eNOS which decreased bladder activity in the same study.

Other animal studies have shown that anticholinergics that can decrease the frequency of urination could increase nNOS expression and decrease iNOS expression in the bladder wall [21]. Significant increased eNOS and nNOS expressions were reported in the bladder of rat with DO induced by BOO too [22]. Although the comparative indicators of bladder function were not exactly the same, these nNOS and iNOS expression changes were different from the findings of our study, and the reasons for this difference are still unclear.

No significant differences in nNOS were found between the presence and absence of DU, although nNOS correlated with the urodynamic parameters related to the bladder contraction in this study. This may be because DU was defined as BCI < 100 in urodynamics. Although this criterion has been used in previous studies $[2,23]$ and in clinical settings, the value of this urodynamic criterion for distinguishing DU remains unclear. In particular, it could be a limitation of urodynamics, as reflected by our findings.

This study had some limitations. We could not include a control group without $\mathrm{BOO}$ in this study because it was difficult to obtain bladder tissue from healthy people. However, men with BOO may have different bladder function status and different values of urodynamic parameters depending on the duration or severity of obstruction. Thus, changes in NOS isoforms can be predicted according to bladder function through a correlation analysis between urodynamic parameters in patients with $\mathrm{BOO}$ and the expression levels of NOS. Our study also had technical limitations, one of which is that specimens contained some part of the lamina propria because it is technically difficult to isolate urothelial tissue without including the lamina propria. There- fore, our findings might include the expression of some NOS isoforms in the lamina propria. Another limitation of our study was that we enrolled a relatively small number of patients that was not reached the target number of sample size in the $\mathrm{BOO}$ with DU group and did not differentiate between the presence and absence of DO. In addition, it is difficult to distinguish whether the change in NO production is due to passive results associated with $\mathrm{BOO}$ or the regulation of $\mathrm{NO}$ production as a biological reaction only by comparing the changes in the expression of NOS. Further studies with more patients are needed to elucidate the relevance of changes in the lower urinary tract function to changes in NOS expression levels over the time in patients with BOO. It can supplement previous studies showing discrepant findings on the relationship between NOS and bladder function, which might be due to the lack of evidence from human studies.

In this study, the relationship between $\mathrm{NO}$ in the urothelium and bladder function was analyzed by assessing the correlation between NOS expression and urodynamic parameters in men with BOO. The results suggest that nNOS is related to detrusor contractility, however we cannot apply this result to actual clinical practice right away. If further research such as identification of the relationship between NOS isoforms expression in the urothelium and in the urine is carried out, we can used NOS isoforms as a simple, less invasive and more accurate diagnostic tool in the clinical practice.

\section{AUTHOR CONTRIBUTION STATEMENT}

\author{
- Conceptualization: JCK \\ - Data curation: JSK \\ - Formal analysis: $J B C$ \\ - Funding acquisition: $K J C$ \\ - Methodology: KJC, SHP, WSL \\ - Project administration: KJC \\ - Visualization: JSK \\ -Writing-original draft: KJC \\ -Writing-review \& editing: KJC
}

\section{ORCID}

$\begin{array}{ll}\text { Kang Jun Cho } & 0000-0002-5305-901 X \\ \text { Jun Sung Koh } & 0000-0001-7432-4209 \\ \text { Jin Bong Choi } & 0000-0003-2508-6819 \\ \text { Joon Chul Kim } & 0000-0002-4019-620 X\end{array}$




\section{REFERENCES}

1. Sutherland RS, Baskin LS, Kogan BA, Cunha G. Neuroanatomical changes in the rat bladder after bladder outlet obstruction. Br J Urol 1998;82:895-901.

2. Hoag N, Gani J. Underactive bladder: clinical features, urodynamic parameters, and treatment. Int Neurourol J 2015;19:185-9.

3. Kim M, Jeong CW, Oh SJ. Effect of preoperative urodynamic detrusor underactivity on transurethral surgery for benign prostatic hyperplasia: a systematic review and meta-analysis. J Urol 2018; 199:237-44.

4. Niemczyk G, Czarzasta K, Radziszewski P, Wlodarski P, CudnochJedrzejewska A. Pathophysiological effect of bladder outlet obstruction on the urothelium. Ultrastruct Pathol 2018;42:317-22.

5. Sellers D, Chess-Williams R, Michel MC. Modulation of lower urinary tract smooth muscle contraction and relaxation by the urothelium. Naunyn Schmiedebergs Arch Pharmacol 2018;391:67594.

6. Kim HS, Kim JC, Choo MS. Effects of nitric oxide synthases on detrusor overactivity after removal of bladder outlet obstruction in rats. Urol Int 2008;81:107-12.

7. Yuan X, Wu S, Lin T, He D, Li X, Liu S, et al. Role of nitric oxide synthase in bladder pathologic remodeling and dysfunction resulting from partial outlet obstruction. Urology 2011;77:1008.e1-8.

8. Drake MJ, Doumouchtsis SK, Hashim H, Gammie A. Fundamentals of urodynamic practice, based on International Continence Society good urodynamic practices recommendations. Neurourol Urodyn 2018;37:S50-60.

9. Cho KJ, Koh JS, Choi J, Kim JC. Changes in adenosine triphosphate and nitric oxide in the urothelium of patients with benign prostatic hyperplasia and detrusor underactivity. J Urol 2017;198:1392-6.

10. Birder L, Andersson KE. Urothelial signaling. Physiol Rev 2013; 93:653-80.

11. Birder LA, Nealen ML, Kiss S, de Groat WC, Caterina MJ, Wang E, et al. Beta-adrenoceptor agonists stimulate endothelial nitric oxide synthase in rat urinary bladder urothelial cells. J Neurosci 2002;22: 8063-70.

12. Sancho M, Ferrero JJ, Triguero D, Torres M, Garcia-Pascual A. Altered neuronal and endothelial nitric oxide synthase expression in the bladder and urethra of cyclophosphamide-treated rats. Nitric Oxide 2014;39:8-19.
13. Jiang YH, Kuo HC. Urothelial barrier deficits, suburothelial inflammation and altered sensory protein expression in detrusor underactivity. J Urol 2017;197:197-203.

14. Felsen D, Dardashti K, Ostad M, Lemer ML, Gross SS, Chen J, et al. Inducible nitric oxide synthase promotes pathophysiological consequences of experimental bladder outlet obstruction. J Urol 2003;169:1569-72.

15. Pereira ML, D’ancona CA, Rojas-Moscoso JA, Ramos AC Filho, Monica FZ, Antunes E. Effects of nitric oxide inhibitors in mice with bladder outlet obstruction. Int Braz J Urol 2017;43:356-66.

16. Biallosterski BT, de Wachter SG, van Koeveringe GA, van Kerrebroeck PE, de Vente J, Mulder MT, et al. Changes in bladder innervation in a mouse model of Alzheimer's disease. J Chem Neuroanat 2010;39:204-10.

17. Hu J, Chin CM, Png JC, Ng YK, Ling EA. The effect of chronic bladder outlet obstruction on neuronal nitric oxide synthase expression in the intramural ganglia of the guinea pig bladder. J Urol 2004;172:1160-5.

18. Hu J, Ng YK, Chin CM, Ling EA. Effects of l-arginine and $\mathrm{N}(\mathrm{G})$ nitro-l-arginine methyl ester treatments on expression of neuronal nitric oxide synthase in the guinea-pig bladder after partial bladder outlet obstruction. Neuroscience 2008;151:680-91.

19. Hannan JL, Powers SA, Wang VM, Castiglione F, Hedlund P, Bivalacqua TJ. Impaired contraction and decreased detrusor innervation in a female rat model of pelvic neuropraxia. Int Urogynecol J 2017;28:1049-56.

20. Theobald RJ Jr. Differing effects of N(G)-monomethyl L-arginine and 7-nitroindazole on detrusor activity. Neurourol Urodyn 2003; 22:62-9.

21. Nishijima S, Sugaya K, Kadekawa K, Ashitomi K, Ueda T, Yamamoto $\mathrm{H}$. Mechanisms underlying the effects of propiverine on bladder activity in rats with pelvic venous congestion and urinary frequency. Biomed Res 2019;40:145-52.

22. Kim SO, Choi D, Song SH, Ahn KY, Kwon D, Park K, et al. Effect of detrusor overactivity on the expression of aquaporins and nitric oxide synthase in rat urinary bladder following bladder outlet obstruction. Can Urol Assoc J 2013;7:E268-74.

23. Osman NI, Chapple CR, Abrams P, Dmochowski R, Haab F, Nitti V, et al. Detrusor underactivity and the underactive bladder: a new clinical entity? A review of current terminology, definitions, epidemiology, aetiology, and diagnosis. Eur Urol 2014;65:389-98. 